\title{
Compact and reflective light-sheet microscopy for long-term imaging of living embryos
}

\author{
BRuno Moretti ${ }^{1,2}$, Nicolás P. MÜLleR ${ }^{1}$, MARCos WAPPNER $^{1}$ AND \\ HERNÁN E. GRECCO ${ }^{1,2, *}$ \\ ${ }^{1}$ Department of Physics, FCEN, University of Buenos Aires, Buenos Aires, Argentina \\ ${ }^{2}$ Instituto de Física de Buenos Aires, CONICET, Buenos Aires, Argentina \\ *hgrecco@df.uba.ar
}

\begin{abstract}
The development of light-sheet fluorescence microscopy has been a revolution for developmental biology as it allows long-term imaging during embryonic development. An important reason behind the quick adoption has been the availability of open hardware alternatives. In this work, we present a robust and compact version of a light-sheet fluorescence microscope (compactLSFM) that is easy to assemble and requires little to no maintenance. An important aspect of the design is that the illumination unit consists of reflective elements, therefore reducing chromatic aberrations an order of magnitude as compared to refractive counterparts.
\end{abstract}

(C) 2019 Optical Society of America under the terms of the OSA Open Access Publishing Agreement

\section{Introduction}

Since its development, fluorescent confocal microscopy has been the tool of choice for imaging biological samples over the last decades [1]. However, imaging large biological structures such as spheroids, organoids and whole embryos over long timescales has proven challenging: observations are limited to around $\sim 100 \mu \mathrm{m}$ from the surface [2], and photobleaching and phototoxicity are typically too high to allow imaging long processes with high temporal resolution [3]. In contrast, light-sheet fluorescence microscopy (LSFM) allows imaging whole, live embryos during timescales of the order of days, with much higher speeds and deeper penetration. Despite the main principle behind this technique has been known for many years [4], it has only recently become popular when it was re-implemented in a fluorescence microscopy version [5]. Open hardware initiatives such as OpenSPIM [6], eduSPIM [7] or SiMView [8] have helped LSFM to get increasingly popular over time, which resulted in the enormous varieties of light-sheet microscopes, both home-made and commercial, that are available today [9].

The optical configuration of the light-sheet microscope diverges from the epi- or transillumination strategy usually found in most optical microscopes. Illumination and imaging objectives are placed perpendicular to each other sharing the focal point. The sample is excited in a thin plane perpendicular to the imaging objective and an image is acquired with a camera, thus achieving two key features of the LSFM: (1) optical sectioning and (2) fast parallel detection. This sheet of light can be achieved by different methods, the simpler one being the use of a cylindrical lens. In this way, LSFM levels of photobleaching and phototoxicity are significantly lower than in confocal microscopy, where the whole volume of the sample is excited for each individual imaging plane. For example, it has been shown that imaging of zebrafish embryos with an LSFM exposed them to 5600 times less energy than a confocal microscope and $10^{6}$ times less energy than a two-photon fluorescence microscope [10]. 
One characteristic most of the non-commercial setups have is the need to have a highly specialized person in charge of constructing, maintaining and operating the microscopes, which hinders access to this technique. Access to a low cost, robust, scientific grade and easy to operate and maintain LSFM would be beneficial to a large community of laboratories that need this technology or require multiplying the number of microscopes to allow high throughput. But simpler implementations are lens-based and therefore subjected to chromatic aberrations. Almost 350 years ago Isaac Newton proposed the following when faced with a similar issue: "the object-glass [...] cannot collect all the rays, which come from one point of an object so as to make them convene at its focus [...] This made me take reflections into consideration." [11]. Following this path, we propose a reflective LSFM system, that it is also compact and robust; and that once installed requires little or no maintenance. We show that the proposed device (named compactLSFM) can be an excellent and affordable addition to laboratories willing to have an accessible and easy to use light-sheet microscope.

\section{Experimental methods}

Our compact light-sheet microscope (Fig. 1a) consists of a laser combination stage, an illumination stage, and a detection stage. To ensure a beam with a Gaussian spatial mode, in the illumination one or more lasers are coupled into a single-mode optical fiber. Although coupling into a fiber produces a significant loss of power, typical coupling efficiencies of around $\sim 10 \%$ for typical laser powers of $\sim 50-100 \mathrm{~mW}$ are usually more than enough for a lightsheet microscope $[5,12,13]$. Having the lasers coupled into a fiber, in addition to ensuring a good spatial mode, has the practical advantage that the light sources are aligned separately from the rest of the microscope, which makes the setup more modular. For example, an additional laser can be added and coupled into the fiber without having to re-align the rest of the microscope. Also, any misalignment in the coupling stage is only traduced in a loss of power, which is not a limiting factor in this setup.

The output of the fiber is sent to a reflective collimator, which has no chromatic aberrations like its refractive counterpart. Using a reflective collimator provides an additional advantage in a fiber-coupled microscope as there is no need to correct the focal distance when a laser with a different wavelength is used. The illumination-dependent axial aberrations are caused by a shift between the light-sheets generated for each wavelength due to slightly different optical paths. Chromatic aberrations in the illumination optics make these detrimental effects more evident. With the same goal of reducing chromatic aberrations, a cylindrical mirror is used to generate a light-sheet instead of a cylindrical lens. The light-sheet is focused on the back focal plane of the illumination objective. The cylindrical mirror is mounted on a right-angle kinematic mirror mount (Thorlabs KCB1/M) that provides three degrees of freedom to align the light-sheet with the illumination objective. This objective focuses the light-sheet in the focal plane of the detection objective.

In our microscope, we use three lasers $(473 \mathrm{~nm}, 532 \mathrm{~nm}$, and $633 \mathrm{~nm})$. For the reflective illumination unit (RIU), we chose a reflective collimator with an output beam diameter of 4 $\mathrm{mm}$ (Thorlabs RC04FC-P01) which in combination with a cylindrical mirror with a focal distance of $50 \mathrm{~mm}$ (Thorlabs CCM254-050-G01), a 0.3 NA illumination objective (Olympus UMPLFLN 10XW), and a 0.5 NA detection objective (Olympus UMPLFLN 20XW) results in an axial resolution of around $7 \mu \mathrm{m}$ (Fig. 1b). The microscope's axial resolution is determined by a combination of the light-sheet thickness, the excitation numerical aperture, and the detection numerical aperture, whereas the lateral resolution is determined by the detection optics [14]. By measuring the axial point spread function (PSF) (see Microscope 
characterization section) we found that the axial resolution is uniform in a field of view of around $0.90 \times 0.76 \mathrm{~mm}$. We do not observe a significant change in lateral and axial PSF upon moving inside the sample. This gives us a field of view big enough to image, for example, Drosophila Melanogaster embryos, which are typically around $500 \mu \mathrm{m}$ long and $250 \mu \mathrm{m}$ thick. For imaging samples with different sizes, the diameter of the light-sheet (and thus the axial resolution), together with the size of the field of view, can be changed by selecting a different reflective collimator. A larger beam diameter will result in a narrower sheet, but also in a bigger divergence, decreasing the effective field of view over which the light-sheet is uniform [14]. This trade-off between light-sheet thickness and field of view size should be kept in mind to select the collimator according to the samples to image. For example, using the Thorlabs RC08FC-P01 collimator would yield a light-sheet about half of the thickness but a 5 times smaller field of view.

The detection path consists of a 0.5 NA objective (Olympus UMPLFLN 20XW) followed by a filter wheel, a tube lens (Thorlabs TTL165-A) and an sCMOS camera (Andor Zyla). Both the illumination and detection objectives are mounted in a $30 \mathrm{~mm}$ cage cube (Thorlabs $\mathrm{C} 4 \mathrm{~W}$ ). To achieve this, each objective is previously mounted on a custom-made part that has an internal O-ring that prevents the mounting media from dripping off the cage cube. Similar parts are designed to cover the remaining cage holes in which no objective is mounted, acting at the same time as inspection windows and allowing to attach an LED for transmission illumination or photoactivation (Fig. 1c). These are the only custom-made parts in the whole setup, the rest being all commercially available. Three-dimensional sample positioning is achieved using three motorized actuators (Newport CMA-12PP) mounted on a translation stage and a universal motion controller (Newport ESP300). Samples are embedded in agarose and mounted on an FEP tube (see Microscope characterization section). This tube is held in the axis of a stepper motor collinear with the microscope's y-axis, allowing sample rotation for multi-view image acquisition. In this way, images can be acquired from several points of view and later computationally fused [15].

Regarding the microscope's temporal resolution, in the end, it will be limited by the components chosen to build the microscope with and by the quality of the fluorescence of the sample being imaged. In our particular case, the camera we use has exposure times as fast as $10 \mathrm{~ms}$, and the limiting factor is the communication speed between the computer controlling the microscope and the motor that scans the samples through the light-sheet. With our microscope, and using exposure times between 10 and $500 \mathrm{~ms}$, it takes between 5 and 30 seconds to scan a distance of $250 \mu \mathrm{m}$ with an axial step of $5 \mu \mathrm{m}$ using a single color.

The filter wheel and shutters used in our device have been developed within an open hardware project (Project SOMA: http://lec.df.uba.ar/soma/). The carcass of the filter wheel and most of its internal parts can be 3D printed, and the wheel is operated with a regular stepper motor controlled by an Arduino board. Similarly, the shutters are constructed with a combination of 3D printed parts and consumer electronics. All the printable files and Arduino software code can be downloaded from the project's website. Finally, the whole microscope is controlled from a Python software (available upon request) based on Lantz (https://github.com/lantzproject) which is also open source. It consists of a graphical interface with several options for automatizing experiments, and due to its modular design, exchanging any of the devices is straight forward. Among other things, the software allows saving arbitrary positions with arbitrary z-scanning parameters to image multiple points of view of the same sample, or to image different samples mounted on the same FEP tube.

\section{Microscope characterization}


To measure the chromatic aberrations of the system, stacks of multi-color fluorescent beads (TetraSpeck Microspheres, $1.0 \mu \mathrm{m}$, fluorescent blue/green/orange/dark red) were imaged using $473 \mathrm{~nm}, 532 \mathrm{~nm}$ and $633 \mathrm{~nm}$ lasers (Fig. 2a). Samples were embedded in agarose and mounted in FEP tubes, with the same procedure we use for living organisms [16]. The stacks were acquired using the same emission filter for the three wavelengths to specifically study chromatic aberrations produced by the excitation part of the microscope. Individual beads from the image (Fig. 2b) were segmented throughout the stack (Fig. 2c) and three-dimensional Gaussians were fitted using PSFj [17]. The coordinates of the center of each bead $\left(x_{c}, y_{c}, z_{c}\right)$ were extracted for each wavelength. The chromatic axial shift was defined as the difference between channels in the value of $z_{c}$. This experiment was repeated using a classical refractive light-sheet generating optics similar to the OpenSPIM microscope [6] without changing the detection optics, so the only variable changed was the excitation part of the microscope. In this classical refractive setup, the same reflective collimator was used, but the light-sheet was generated using a cylindrical lens (Thorlabs ACY254-050-A). The light-sheet was focused on a corner mirror, and a telescope composed of two lenses (Thorlabs AC254-030-A-ML and AC254-060-A-ML) was used to conjugate the light-sheet on the back focal plane of the illumination objective.

As can be seen in the comparison of the axial shifts between both setups (Fig. 2d), using reflective illumination optics reduces chromatic aberrations significantly. To quantify the global axial shift, the median values of the distributions corresponding to each pair of channels were calculated. The statistical errors were defined as the standard deviation over the number of beads analyzed in each case. For the reflective setup, the average axial shift found was negligible $(10.2 \pm 50.6) \mathrm{nm}$ between $473 \mathrm{~nm}$ and $532 \mathrm{~nm}$ lasers, $(54.4 \pm 12.4) \mathrm{nm}$ between 532 $\mathrm{nm}$ and $633 \mathrm{~nm}$ lasers, and $(91.7 \pm 17.2) \mathrm{nm}$ between $473 \mathrm{~nm}$ and $633 \mathrm{~nm}$ lasers. For the classical refractive setup, we measured an average shift of $(210 \pm 155) \mathrm{nm}$ between $473 \mathrm{~nm}$ and $532 \mathrm{~nm}$ lasers, $(766 \pm 52) \mathrm{nm}$ between $532 \mathrm{~nm}$ and $633 \mathrm{~nm}$ lasers, and $(980 \pm 57) \mathrm{nm}$ between $473 \mathrm{~nm}$ and $633 \mathrm{~nm}$ lasers. The differences between these shifts in the refractive and reflective setups are statistically significant according to a Kolmogorov-Smirnov test in all cases. Being determined by the detection optics, the lateral resolution and aberrations were not changed by using the reflective illuminator unit and resulted in comparable values to those previously reported $[6,17]$.

\section{Application examples}

We chose to demonstrate our compactLSFM by imaging Drosophila embryos. Briefly, all samples were prepared from crosses that were made according to need in bottles with Petri dishes with agar, sucrose, and yeast as lids. Flies lay eggs on the agar, and those eggs are later collected and dechorionated using bleach. Dechorionated embryos are transferred to a tube containing low melting point agarose and pulled up inside an FEP tube using a syringe. Stacks of 75 images were acquired with an axial step of $2 \mu \mathrm{m}$.

This procedure enables us to observe samples from different points of view, allowing us to image the dorsal and ventral sides of Drosophila embryos, that are $180^{\circ}$ apart from each other. To demonstrate this we imaged an embryo from a transgenic line expressing a FRET caspase activity sensor [18,19] at stage 10 (Fig. 3a). The exposure time was $20 \mathrm{~ms}$ for both points of view, for each plane. As can be seen, the contrast is good enough to segment and track individual nuclei if a timelapse is acquired with sufficient temporal resolution. Using an opensource image processing software [20] it is also possible to obtain a three-dimensional reconstruction as shown for a stage 13 His-RFP embryo (Fig. 3b). The exposure time was 50 ms for each plane. On the left side of the embryo, the open amnioserosa can be clearly seen, 
and on the right side, the already developed embryo segments are visible. Snapshots of a timelapse from the same sample show the embryo undergoing germ band retraction, segment formation and dorsal closure (Fig. 3c). Individual slices of a region of the embryo at different depths are shown (Fig. 3d).

\section{Conclusions}

In this work, we have presented a compact and reflective light-sheet fluorescence microscope, suitable for long-term imaging of living embryos. We have demonstrated the imaging capabilities of this novel device by imaging Drosophila embryos during development. Microscopes with similar optical characteristics have been used to image different organisms such as Medaka and Zebrafish embryos, among others. Therefore, we expect that the design presented here could be used by those communities as well. Our design is affordable, robust and does not require any further alignment after it is built, making it ideal for a large number of laboratories that require daily access to such imaging devices. The optical characteristics are comparable to similar refractive LSFM but the chromatic aberrations are an order of magnitude smaller. Due to its modular design, adding a second detection or illumination arm as has been done for other open hardware microscopes can be easily achieved. We expect that this novel and affordable design makes multicolor LSFM even more accessible to laboratories around the world.

\section{Funding}

Fondo para la Investigación Científica y Tecnológica (2013-1301,2014-3658), Max-PlanckGesellschaft (Partner Group), Secretaría de Ciencia y Técnica (20020170100755BA)

\section{Disclosures}

The authors declare no conflicts of interest.

\section{References}

1. J. Jonkman and C. M. Brown, "Any way you slice it-A comparison of confocal microscopy techniques," J. Biomol. Tech. 26, 54-65 (2015).

2. C. L. Smithpeter, A. K. Dunn, A. J. Welch, and R. Richards-Kortum, "Penetration depth limits of in vivo confocal reflectance imaging," Conf. Proc. - Lasers Electro-Optics Soc. Annu. Meet. 11, 42 (1997).

3. M. Jemielita, M. J. Taormina, A. DeLaurier, C. B. Kimmel, and R. Parthasarathy, "Comparing phototoxicity during the development of a zebrafish craniofacial bone using confocal and light sheet fluorescence microscopy techniques," J. Biophotonics 6, 920-928 (2013).

4. H. Siedentopf and R. Zsigmondy, "Uber sichtbarmachung und größenbestimmung ultramikoskopischer teilchen, mit besonderer anwendung auf goldrubingläser," Ann. der Phys. (1902).

5. J. Huisken, J. Swoger, F. Del Bene, J. Wittbrodt, and E. H. K. Stelzer, "Optical Sectioning Deep Inside Live Embryos by Selective Plane Illumination Microscopy," Science (80-. ). 305, 1007-1009 (2004).

6. P. G. Pitrone, J. Schindelin, L. Stuyvenberg, S. Preibisch, M. Weber, K. W. Eliceiri, J. Huisken, and P. Tomancak, "OpenSPIM: an open-access light-sheet microscopy platform.," Nat. Methods 10, 598-9 (2013).

7. W. Jahr, B. Schmid, M. Weber, and J. Huisken, "eduSPIM : Light Sheet Microscopy in the Museum," $1-$ 12 (2016).

8. L. A. Royer, W. C. Lemon, R. K. Chhetri, and P. J. Keller, "A practical guide to adaptive light-sheet microscopy," Nat. Protoc. 13, 2462-2500 (2018).

9. J. Huisken and D. Y. R. Stainier, "Selective plane illumination microscopy techniques in developmental biology.," Development 136, 1963-1975 (2009).

10. P. J. Keller, A. D. Schmidt, J. Wittbrodt, and E. H. K. Stelzer, "Reconstruction of zebrafish early embryonic development by scanned light sheet microscopy.," Science 322, 1065-1069 (2008). 
11. I. Newton, "A letter of Mr. Isaac Newton, Professor of the Mathematicks in the University of Cambridge; containing his new theory about light and colors: sent by the author to the publisher from Cambridge, Febr. 6. 1671/72; in order to be communicated to the R. Socie," Philos. Trans. R. Soc. London 6, 3075-3087 (1671).

12. J. G. Ritter, R. Veith, J.-P. Siebrasse, and U. Kubitscheck, "High-contrast single-particle tracking by selective focal plane illumination microscopy.," Opt. Express 16, 7142-7152 (2008).

13. H.-U. Dodt, U. Leischner, A. Schierloh, N. Jährling, C. P. Mauch, K. Deininger, J. M. Deussing, M. Eder, W. Zieglgänsberger, and K. Becker, "Ultramicroscopy: three-dimensional visualization of neuronal networks in the whole mouse brain.," Nat. Methods 4, 331-336 (2007).

14. L. Gao, L. Shao, B.-C. Chen, and E. Betzig, "3D live fluorescence imaging of cellular dynamics using Bessel beam plane illumination microscopy.," Nat. Protoc. 9, 1083-101 (2014).

15. S. Preibisch, S. Saalfeld, J. Schindelin, and P. Tomancak, "Software for bead-based registration of selective plane illumination microscopy data.," Nat. Methods 7, 418-9 (2010).

16. A. Kaufmann, M. Mickoleit, M. Weber, and J. Huisken, "Multilayer mounting enables long-term imaging of zebrafish development in a light sheet microscope," Development 139, 3242-3247 (2012).

17. P. Theer, C. Mongis, and M. Knop, "PSFj: Know your fluorescence microscope," Nat. Methods 11, $981-$ $982(2014)$

18. L. Stegemann, K. C. Schuermann, C. A. Strassert, and H. E. Grecco, "Photofunctional Surfaces for Quantitative Fluorescence Microscopy: Monitoring the Effects of Photogenerated Reactive Oxygen Species at Single Cell Level with Spatiotemporal Resolution," ACS Appl. Mater. Interfaces 7, 5944-5949 (2015).

19. A. A. Corbat, K. C. Schuermann, P. Liguzinski, Y. Radon, P. I. H. Bastiaens, P. J. Verveer, and H. E. Grecco, "Co-imaging extrinsic, intrinsic and effector caspase activity by fluorescence anisotropy microscopy," (2018).

20. F. De Chaumont, S. Dallongeville, N. Chenouard, N. Hervé, S. Pop, T. Provoost, V. Meas-Yedid, P. Pankajakshan, T. Lecomte, Y. Le Montagner, T. Lagache, A. Dufour, and J. C. Olivo-Marin, "Icy: An open bioimage informatics platform for extended reproducible research," Nat. Methods 9, 690-696 (2012). 
(a)

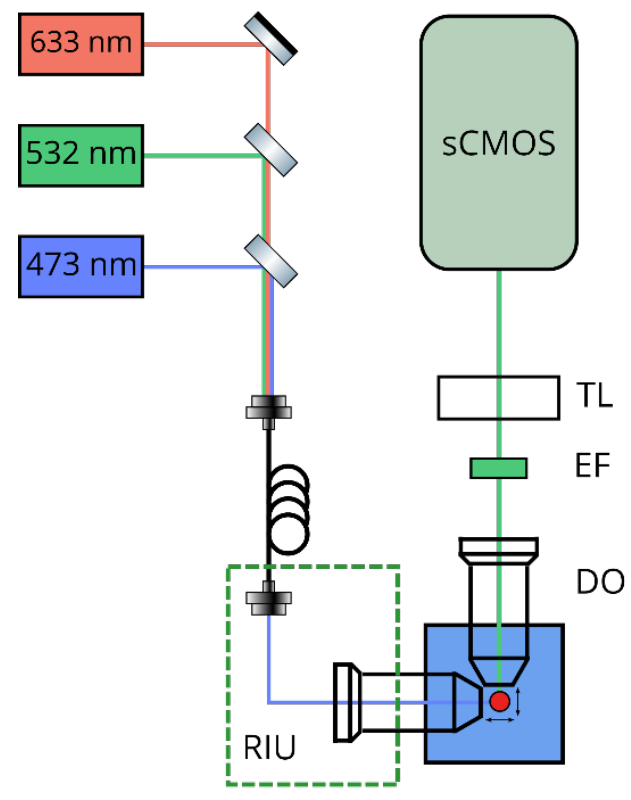

(c)

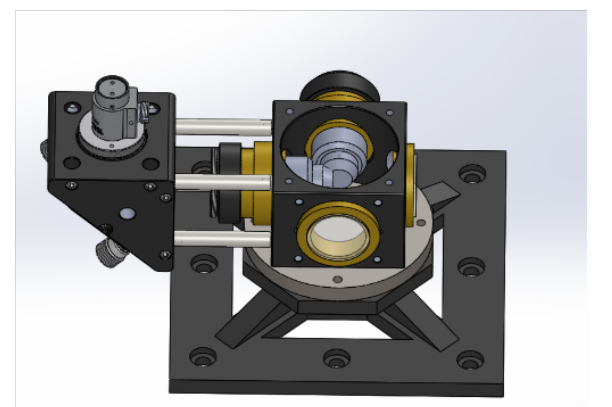

(b)

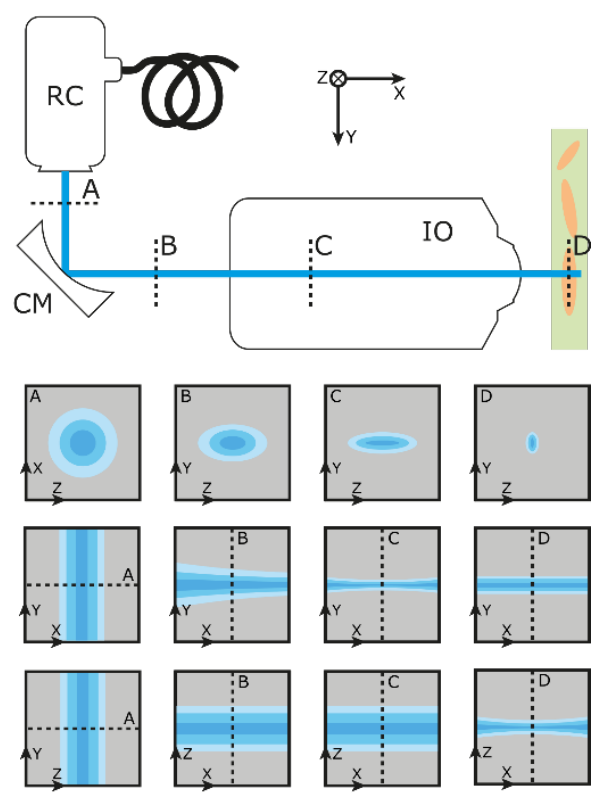

(d)

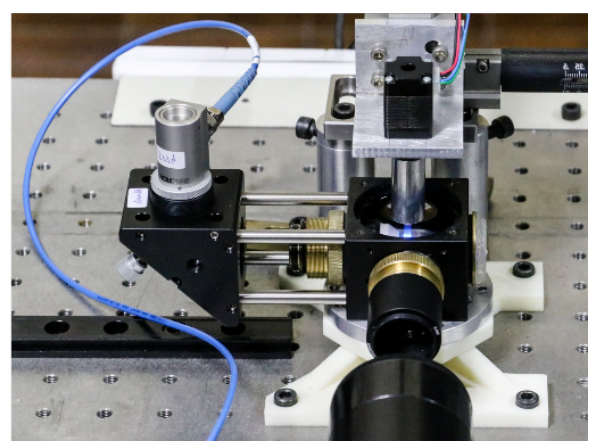

Figure 1: Diagram of the compact light-sheet microscope. (a) Three lasers are coupled into a single-mode optical fiber. The illumination stage consists of a reflective collimator RC followed by a reflective illumination unit RIU detailed in b. In the detection stage, a detection objective DO is followed by an emission filter EM, a tube lens TL and an sCMOS camera. (b) Top: Detail of the reflective illumination unit. A reflective collimator RC generates a collimated Gaussian beam that is reflected by a cylindrical mirror $\mathbf{C M}$. The cylindrical mirror generates a horizontal sheet of light propagating in the $x$-direction that is focused on the $y$-direction and collimated in the $z$-direction. The light-sheet is focused on the back focal plane of the illumination objective IO, which changes the plane of focus from $x-y$ to $x-z$. Samples are later positioned in the light-sheet waist and scanned through it. Bottom: illustrations of the changes in the shape of the beam when going through the different optical components of the microscope, in the $x-y$, $x-z$ and $y-z$ planes at different relevant points in the RIU. It is worth noting that in the $y$-direction the cylindrical mirror focuses the beam at the back focal plane of the objective (C), which then, in turn, is collimated by it to reach the sample (D). In the $z$-direction, the opposite occurs: the cylindrical mirror does not focus the beam from the collimator (A vs B and C) which is then focused by the objective in the sample (D) (c) 3D render showing the reflective collimator, corner cylindrical mirror, illumination and detection objectives, and imaging chamber. (d) Picture of compactLSFM. The fiber-coupled RIU (left) is fixed to the imaging chamber with metallic rods. To the front, $90^{\circ}$ from the illumination objective is the detection objective. The sample holder is maintained vertically, in the axis of a stepper motor, to allow sample rotation. (The $x-y-z$ motorized stage, filter wheel, tube lens, and camera, which are standard, are not shown). 
(a)

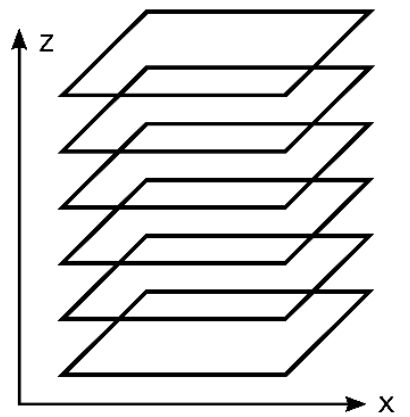

(b)

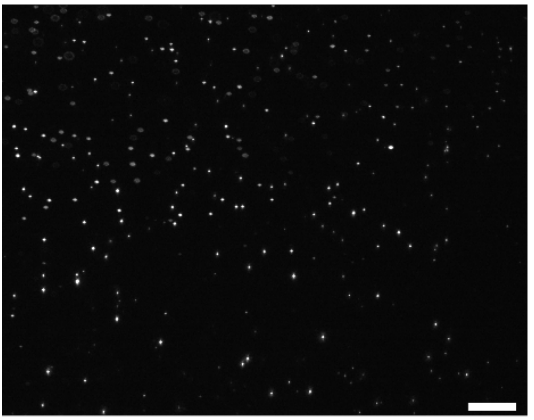

Single bead

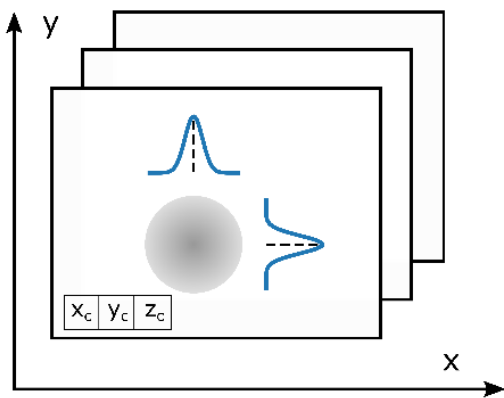

(d)

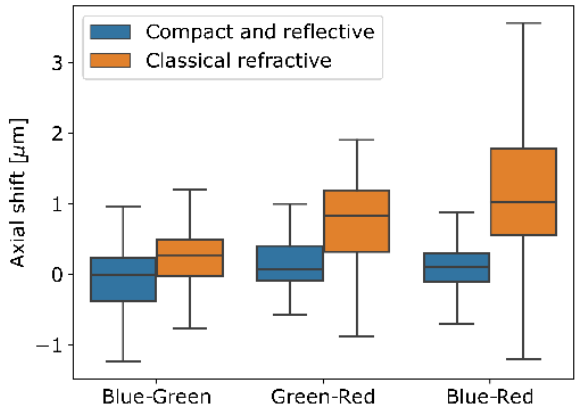

Figure 2: Characterization of chromatic aberrations (a) Diagram of the workflow for measuring the microscope's axial shift. Stacks of multi-color fluorescent beads are acquired across the $z$-direction. Three-dimensional Gaussian functions are fitted to individual beads using PSFj and the centers of the beads $\left(x_{c}, y_{c}, z_{c}\right)$ are extracted. The shift between different channels in the axial coordinate of the center of the bead $z_{c}$ is a measure of the microscope's axial chromatic aberrations. (b) Example of a single z-slice of multi-color fluorescent beads acquired with the compact lightsheet microscope. Scale bar: $50 \mu \mathrm{m}$ (c) Close-up of the image of a single bead across z. Scale bar: $5 \mu \mathrm{m}$ (d) Box plot of the axial shift between different channels corresponding to blue $(473 \mathrm{~nm})$, green $(532 \mathrm{~nm})$ and red $(633 \mathrm{~nm})$ illumination wavelengths. This measurement was repeated for a refractive setup of similar conditions, showing that in the reflective microscope chromatic aberrations are significantly lower. The horizontal lines correspond to the median values of each distribution, and the points corresponding to quartiles 1-3 (25th to 75th percentile) are plotted inside the box. The whiskers represent the maximum and minimum values, without considering outliers. The number of beads $\mathrm{N}$ analyzed for the reflective setup was $\mathrm{N}=91$ for the blue-green channels, $\mathrm{N}=56$ for the green-red channels, and $\mathrm{N}=171$ for the blue-red channels. For the classical refractive setup, $\mathrm{N}=193$ beads were analyzed for the green-blue channels, $\mathrm{N}=207$ for the green-red channels, and N=184 for the blue-red channels. P-values from Kolmogorov-Smirnov statistical tests are less than 0.01 for all cases. 

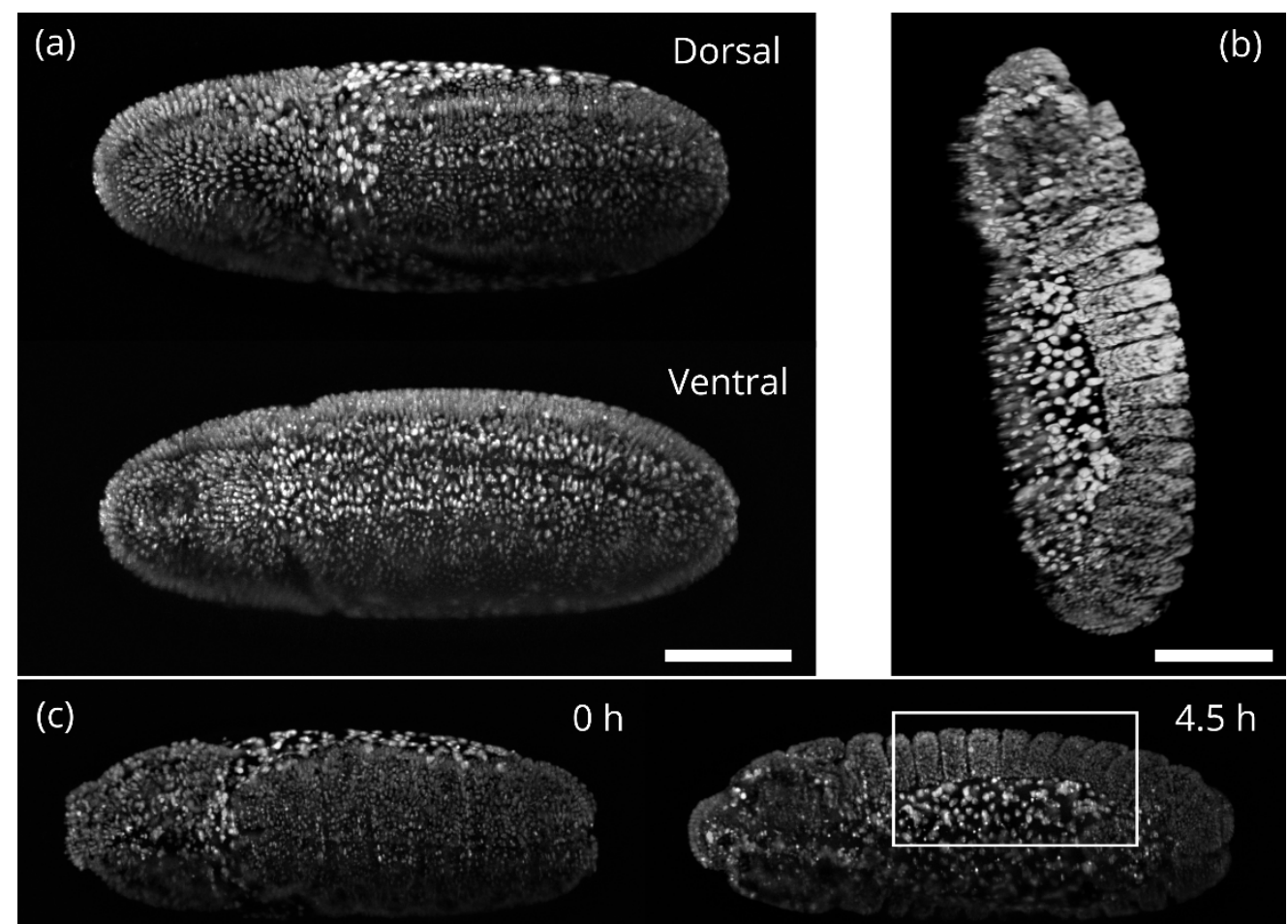

$7.8 \mathrm{~h}$

$10.5 \mathrm{~h}$
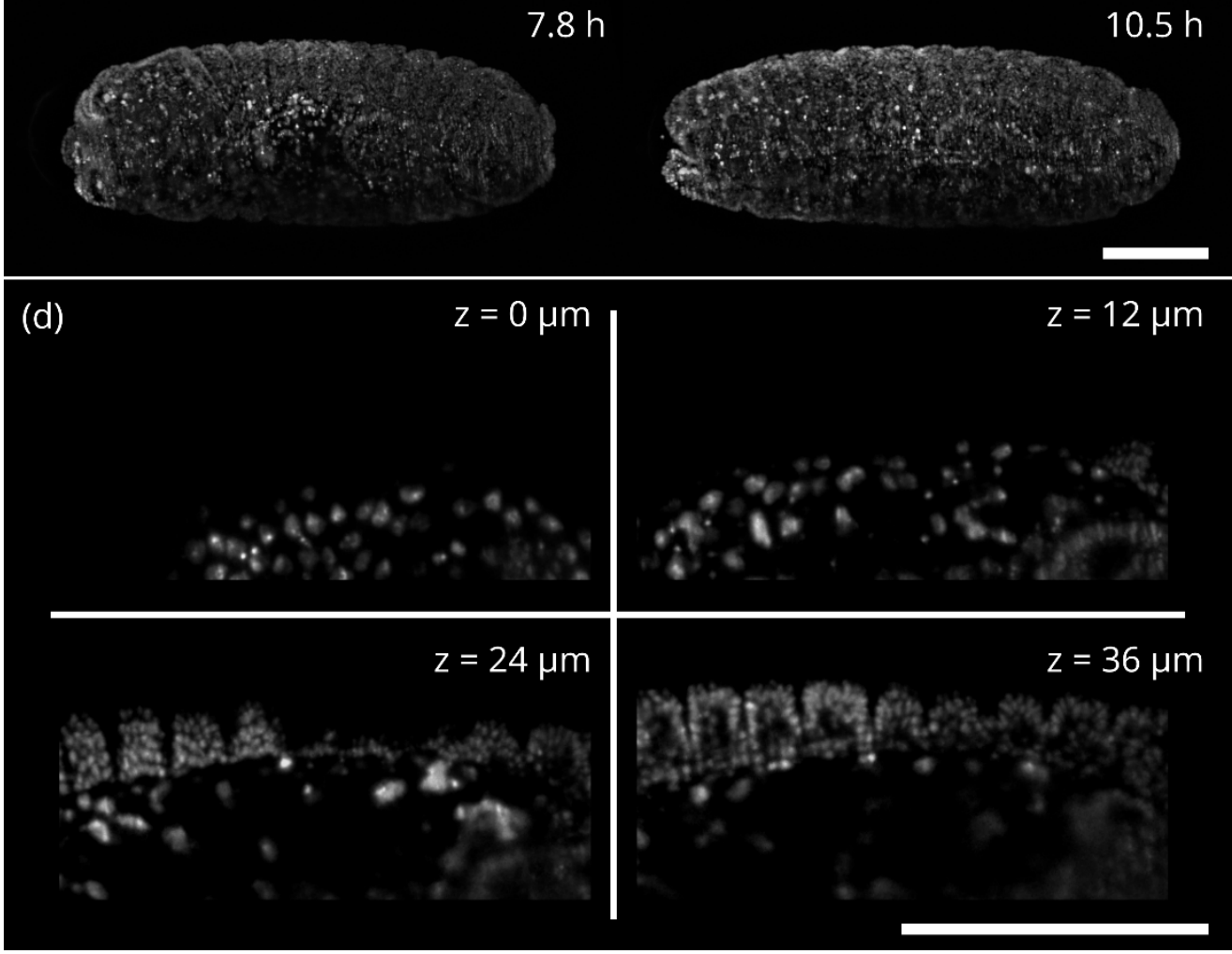

Figure 3: Application example (a) Drosophila embryo imaged from the dorsal and ventral sides with our compact light-sheet microscope. Maximum intensity projections of 3D stacks are shown. The embryo expresses a FRET sensor 
driven by Tubulin. The exposure time used was $20 \mathrm{~ms}$. (b) 3D reconstruction of a stage 13 Drosophila embryo expressing His-RFP. The bigger amnioserosa cells can be seen on the left side of the image, and the already developed segments on the right side. The exposure time was $50 \mathrm{~ms}$. (c) Developing His-RFP Drosophila embryo imaged from a dorsolateral view. The exposure time used was $50 \mathrm{~ms}$. The first frame is at stage 11, with the germ band fully extended. $4.5 \mathrm{~h}$ later the germ band has retracted and amnioserosa is exposed. At $7.8 \mathrm{~h}$ after the first frame the embryo is undergoing dorsal closure, and at $10.5 \mathrm{~h}$ dorsal closure has fully concluded. (d) Detail of slices at different depths of the region highlighted by the region of interest in (c). The axial distance between the slices shown is $12 \mu \mathrm{m}$. Scale bar in all panels: $100 \mu \mathrm{m}$. 